\title{
Hot shot induction and reperfusion with a specific blocker of the es-ENT1 nucleoside transporter before and after hypothermic cardioplegia abolishes myocardial stunning in acutely ischemic hearts despite metabolic derangement: Hot shot drug delivery before hypothermic cardioplegia
}

\author{
Anwar Saad Abd-Elfattah, PhD, ${ }^{a}$ Gert E. Tuchy, MD, ${ }^{\text {a }}$ Michael E. Jessen, MD, ${ }^{\mathrm{b}}$ David R. Salter, MD, ${ }^{\mathrm{a}}$
} Jacques P. Goldstein, MD, PhD, ${ }^{c}$ Louis A. Brunsting III, MD, ${ }^{d}$ and Andrew S. Wechsler, MD ${ }^{\mathrm{e}}$

\begin{abstract}
Objective: Simultaneous inhibition of the cardiac equilibrative-p-nitrobenzylthioinosine (NBMPR)-sensitive (es) type of the equilibrative nucleoside transport 1 (ENT1) nucleoside transporter, with NBMPR, and adenosine deaminase, with erythro-9-[2-hydroxy-3-nonyl]adenine (EHNA), prevents release of myocardial purines and attenuates myocardial stunning and fibrillation in canine models of warm ischemia and reperfusion. It is not known whether prolonged administration of hypothermic cardioplegia influences purine release and EHNA/NBMPR-mediated cardioprotection in acutely ischemic hearts.
\end{abstract}

\begin{abstract}
Methods: Anesthetized dogs $(n=46)$, which underwent normothermic aortic crossclamping for 20 minutes onpump, were divided to determine (1) purine release with induction of intermittent antegrade or continuous retrograde hypothermic cardioplegia and reperfusion, (2) the effects of postischemic treatment with $100 \mu \mathrm{M}$ EHNA and $25 \mu \mathrm{M}$ NBMPR on purine release and global functional recovery, and (3) whether a hot shot and reperfusion with EHNA/NBMPR inhibits purine release and attenuates ventricular dysfunction of ischemic hearts. Myocardial biopsies and coronary sinus effluents were obtained and analyzed using high-performance liquid chromatography.

Results: Warm ischemia depleted myocardial adenosine triphosphate and elevated purines (ie, inosine $>$ adenosine) as markers of ischemia. Induction of intermittent antegrade or continuous retrograde hypothermic $\left(4^{\circ} \mathrm{C}\right)$ cardioplegia releases purines until the heart becomes cold $\left(<20^{\circ} \mathrm{C}\right)$. During reperfusion, the levels of hypoxanthine and xanthine (free radical substrates) were $>90 \%$ of purines in coronary sinus effluent. Reperfusion with EHNA/NBMPR abolished ventricular dysfunction in acutely ischemic hearts with and without a hot shot and hypothermic cardioplegic arrest.
\end{abstract}

Conclusions: Induction of hypothermic cardioplegia releases purines from ischemic hearts until they become cold, whereas reperfusion induces massive purine release and myocardial stunning. Inhibition of cardiac es-ENT1 nucleoside transporter abolishes postischemic reperfusion injury in warm and cold cardiac surgery. (J Thorac Cardiovasc Surg 2013;146:961-70)

\section{Supplemental material is available online.}

\footnotetext{
From the Cardiovascular Research Laboratories, ${ }^{\text {a }}$ Division of Cardiothoracic Surgery, Department of Surgery, VCU Medical Center, Richmond, Va; the University of Texas South Western, ${ }^{\mathrm{b}}$ Dallas, Tex; the Department of Cardiac Surgery, ${ }^{\mathrm{c}}$ Academic Hospital Vrije Universiteit Brussel, Brussels, Belgium; the Centennial Medical Center, ${ }^{\mathrm{d}}$ Vanderbilt University, Nashville, Tenn; and Drexel University Medical Center, ${ }^{\mathrm{e}}$ Philadelphia, Pa.

Supported in part by the National Institute of Health (NIH) to A. S. Abd-Elfatta (grant HL 5-1090) and A. S. Wechsler (grant HL-26302).

Disclosures: Authors have nothing to disclose with regard to commercial support.

Received for publication July 1, 2012; revisions received Sept 27, 2012; accepted for publication Oct 16, 2012; available ahead of print Feb 19, 2013.

Address for reprints: Anwar Saad Abd-Elfattah, PhD, Cardiothoracic Surgery, Department of Surgery, PO Box 980068, Richmond, VA 23298-0068 (E-mail: anwar@vcu.edu)

0022-5223/\$0.00

Published by Elsevier Inc. on behalf of The American Association for Thoracic Surgery

http://dx.doi.org/10.1016/j.jtcvs.2012.10.054
}

Patients who present for thrombolytic therapy, percutaneous coronary interventions, and coronary artery bypass grafting experience variable degrees of metabolic derangement and functional and electrophysiologic abnormalities prior to cardiac surgery. Although resumption of blood flow (reperfusion) to ischemic myocardium alleviates oxidative stress and salvages the myocardium, it promotes vascular damage and ventricular dysfunction (myocardial stunning). ${ }^{1-4}$ Reperfusion injury is triggered initially by the production of oxygen-derived free radicals, ${ }^{2,4,5}$ whereas activation of neutrophils ${ }^{6}$ and inflammatory responses ${ }^{7}$ play a role in late consequences of reperfusion injury.

The prolonged intraoperative recovery period makes the heart more vulnerable to arrhythmias, fibrillation, and pump failure, and affects adversely the outcome of cardiac surgery. Developing new modalities that manage reperfusion injury is a reasonable approach toward cardioprotection in different clinical settings of ischemic 


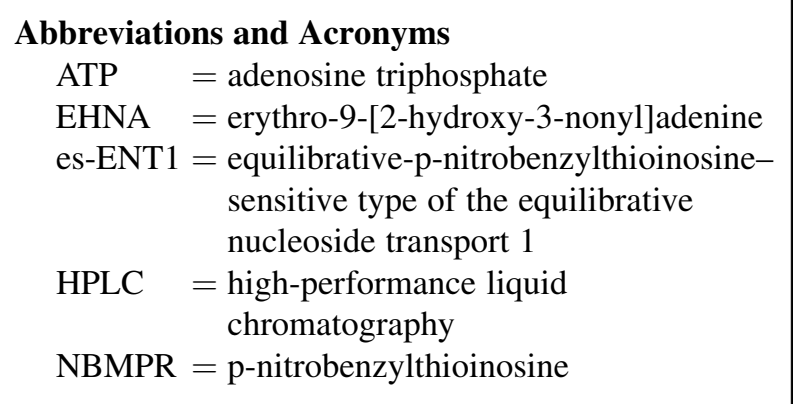

syndromes. For the past 3 decades, ischemic preconditioning maneuvers applied on naive hearts and normal animals provided powerful cardioprotective responses, yet they failed to protect further stunned, ${ }^{8,9}$ infarcted, ${ }^{10}$ hypertrophied, and failing hearts. ${ }^{11,12}$ Therefore, well-defined and specific pharmacologic interventions capable of preventing ventricular dysfunction or accelerating functional recovery of stunned, infarcted, hypertrophied, and failing hearts undergoing cardiac surgery assume significant clinical relevance.

Previously, we have shown that normothermic global or regional ischemia promotes purine release, and that it was associated with sustained ventricular dysfunction and increased the incidents of ventricular fibrillation. ${ }^{2-4,8,9,13-15}$ Furthermore, we demonstrated that pre- and postischemic inhibition of the equilibrative-p-nitrobenzylthioinosine (NBMPR)-sensitive (es) type of the equilibrative nucleoside transport 1 (ENT1) nucleoside transport with NBMPR and adenosine deaminase activity with erythro9-[2-hydroxy-3-nonyl]adenine (EHNA) blocks release of purines and attenuates ventricular dysfunction and fibrillation. ${ }^{2-4,8,9,13-15}$ The inhibitory effects of EHNA/NBMPR are site specific and are limited to the ischemic myocardium. ${ }^{4}$ Although we have demonstrated the efficacy of EHNA/NBMPR intervention in various models of warm ischemia and reperfusion, it is not known whether prolonged infusion of hypothermic cardioplegia affects purine release from ischemic hearts and subsequently influences cardioprotection offered by EHNA/NBMPR treatment.

The goals of the current study are (1) to determine whether infusion of intermittent antegrade or continuous retrograde hypothermic cardioplegia into acute ischemic hearts triggers releases of adenosine triphosphate (ATP) catabolites, which are known to be markers of ischemia and substrates for the production of oxygen-derived free radicals on reperfusion; and (2) to determine the role of the NBMPR-sensitive es-ENT1 nucleoside transporter in reperfusion injury in acutely ischemic hearts undergoing either unprotected warm ischemia and reperfusion or warm ischemia followed by prolonged hypothermic cardioplegic arrest and reperfusion.

\section{METHODS \\ Materials}

Biochemical reagents, EHNA, and NBMPR were purchased from Sigma-Aldrich Chemical Company (St. Louis, Mo).

\section{Animal Model}

The following studies conform to the Guide for the Care and Use of Laboratory Animals published by the US National Institute of Health. ${ }^{16}$

Forty-six microfilaria-free adult mongrel dogs of either sex, weighing 18 to $26 \mathrm{~kg}$ were used in this study. Dogs were anesthetized initially with $35 \mathrm{mg} / \mathrm{kg}$ sodium pentobarbital intravenously (Nembutal; Abbott Laboratories, Chicago, Ill) and maintained with $10-\mathrm{mg} / \mathrm{kg}$ boluses of sodium pentobarbital. The surgical procedure and instrumentation were performed as described previously. ${ }^{2}$ The right phrenic nerve was transected and the sinoatrial node was crushed, and the heart was paced at rate of $150 \mathrm{bpm}$. Body and heart temperatures were maintained at $37 \pm 1^{\circ} \mathrm{C}$. Dogs were heparinized with an intravenous injection with porcine-based heparin $(400 \mathrm{U} / \mathrm{kg}$ ) as an initial bolus followed by $200 \mathrm{U} / \mathrm{kg} / \mathrm{hour}$ (Elkins-Sinn Inc, Cherry Hill, NJ), then placed on cardiopulmonary bypass using a membrane oxygenator (Medtronic, Minneapolis, Minn). The extracorporeal circulation was primed with noncross-matched homologous blood from a bleeder dog, and an aortic cannula and a 2-stage venous cannula were introduced into the right atrium and the inferior vena cava, respectively. The azygous vein was occluded and the left ventricle was vented at the apex. The mean arterial reperfusion pressure was maintained at 60 to $65 \mathrm{~mm} \mathrm{Hg}$ while on bypass. Arterial blood gases, pH, and hematocrit were determined routinely and maintained at the following levels: pressure of oxygen, $100-140 \mathrm{~mm} \mathrm{Hg}$; pressure of carbon dioxide, $30-40 \mathrm{~mm} \mathrm{Hg}$; pH, 7.32 to 7.48 ; and hematocrit, about $30 \%$.

All dogs underwent 20 minutes of normothermic aortic crossclamping on-pump, during which a $12 \mathrm{~F}$ Foley catheter was introduced into the coronary sinus orifice under direct vision via right atriotomy. Potassiumbased cardioplegic solution containing $25 \mathrm{~g} / \mathrm{L}$ dextrose, $110 \mathrm{mEq} / \mathrm{L} \mathrm{Na}^{+}$, $162 \mathrm{mEq} / \mathrm{L} \mathrm{Cl}^{-}, 1.0 \mathrm{mEq} / \mathrm{L} \mathrm{Ca}^{2+}, 32 \mathrm{mEq} / \mathrm{L} \mathrm{Mg}^{2+}, 16 \mathrm{mEq} / \mathrm{L} \mathrm{K}^{+}, 0.98$ $\mathrm{mmol} / \mathrm{L}$ procaine, and Tris buffer $(\mathrm{pH} 7.8)$ at $4{ }^{\circ} \mathrm{C}$ with an osmolarity of $419 \mathrm{mOsm} / \mathrm{L}$ was infused with either an intermittent antegrade (via aortic) cannula (1000-mL initial bolus plus 500-mL boluses every 20 minutes thereafter via an aortic cannula) or as a continuous retrograde coronary sinus infusion through the Foley catheter for 100 minutes at a maximal pressure of $40 \mathrm{~mm} \mathrm{Hg}$ (20-25 mL/minute). Antegrade cardioplegia was collected from the coronary sinus whereas the retrograde cardioplegic solution washout was collected via an aortic cannula.

Dogs were divided into 3 experimental protocols as described in Figure E1. The first protocol was established to determine whether infusion of intermittent antegrade ( $n=7$ dogs) or continuous retrograde $(n=7$ dogs $)$ hypothermic cardioplegia, following 20 minutes of warm aortic crossclamping, promotes the release of myocardial purines from ischemic hearts. In these experiments, the infused cardioplegic solution was collected from the coronary sinus cannula in the intermittent antegrade group or from the aortic root in the continuous retrograde group. Topical cooling was used to protect the right ventricle. Transmural myocardial biopsies were collected at baseline, at the end of normothermic aortic crossclamping, during 100 minutes of infusion of hypothermic cardioplegia, and during 60 minutes of reperfusion.

The second protocol was established to determine the effects of postischemic administration of EHNA/NBMPR on myocardial purines and recovery of ventricular performance without a period of hypothermic cardioplegic arrest. Hearts were subjected to 20 minutes of warm aortic crossclamping followed by reperfusion with warm blood containing the vehicle solution $(\mathrm{n}=8)$ or the vehicle solution with $25 \mu \mathrm{M}$ NBMPR and $100 \mu$ M EHNA $(\mathrm{n}=8)$. A bolus of $1000 \mathrm{~mL}$ of the vehicle or the vehicle solution containing $100 \mu \mathrm{M}$ EHNA and $25 \mu \mathrm{M}$ NBMPR was infused into the pump 5 minutes prior to reperfusion. Myocardial biopsies were obtained at baseline, during cold cardioplegic arrest, and during reperfusion. 
TABLE 1. Effects of intermittent antegrade or continuous coronary sinus retrograde hypothermic cardioplegia on myocardial purine levels in acutely ischemic hearts

\begin{tabular}{|c|c|c|c|c|c|c|c|c|}
\hline \multirow[b]{2}{*}{ Purine } & \multirow[b]{2}{*}{ Preischemia } & \multirow[b]{2}{*}{ Ischemia, $20 \mathrm{~min}$} & \multicolumn{4}{|c|}{ Hypothermic cardioplegia } & \multicolumn{2}{|c|}{ Reperfusion } \\
\hline & & & $10 \mathrm{~min}$ & 55 min & 85 min & $99 \mathrm{~min}$ & $30 \mathrm{~min}$ & $60 \mathrm{~min}$ \\
\hline ATP-AC & $35.7 \pm 3.7$ & $26.0 \pm 2.0^{*}$ & $31.0 \pm 1.6$ & $26.2 \pm 2.7$ & $28.6 \pm 3.2$ & $28.5 \pm 2.3$ & $22.2 \pm 1.2^{*}$ & $21.6 \pm 2.6^{*}$ \\
\hline ATP-RCSC & $27.0 \pm 2.4$ & $21.3 \pm 1.2^{*}$ & $27.3 \pm 2.7$ & $27.1 \pm 4.0$ & $27.3 \pm 3.1$ & $25.4 \pm 2.6$ & $22.6 \pm 2.6^{*}$ & $21.4 \pm 2.1^{*}$ \\
\hline INO-AC & $0.1 \pm 0.1$ & $7.8 \pm 1.0^{*}$ & $8.3 \pm 0.9^{*}$ & $7.6 \pm 1.0^{*}$ & $9.2 \pm 1.9^{*}$ & $7.2 \pm 1.2^{*}$ & $0.2 \pm 0.1$ & $0.3 \pm 0.1$ \\
\hline INO-RCSC & $0.1 \pm 0.1$ & $6.7 \pm 1.4^{*}$ & $9.9 \pm 1.4^{*}$ & $9.8 \pm 1.4^{*}$ & $8.7 \pm 1.0^{*}$ & $8.5 \pm 1.6^{*}$ & $0.1 \pm 0.1$ & $0.1 \pm 0.1$ \\
\hline TDP-AC & $1.2 \pm 0.3$ & $12.0 \pm 1.4^{*}$ & $11.9 \pm 1.1^{*}$ & $10.5 \pm 1.0^{*}$ & $11.3 \pm 2.3^{*}$ & $10.5 \pm 1.4^{*}$ & $1.3 \pm 0.1$ & $1.2 \pm 0.5$ \\
\hline TDP-RCSC & $1.4 \pm 0.5$ & $10.1 \pm 1.1^{*}$ & $13.8 \pm 1.5^{*}$ & $13.1 \pm 1.9^{*}$ & $12.0 \pm 1.2^{*}$ & $11.6 \pm 2.3^{*}$ & $1.3 \pm 0.1$ & $1.7 \pm 0.3$ \\
\hline
\end{tabular}

Purine levels are presented as moles per milligram protein, mean \pm standard error of the mean. $P=$ not significant, 2 -way analysis of variance. $A T P$, Adenosine triphosphate; $A C$, antegrade cardioplegia; $R C S C$, retrograde coronary sinus cardioplegia; $I N O$, inosine; $T D P$, total diffusible purines (adenosine + inosine + hypoxanthine + xanthine). $* P<.05$ versus corresponding preischemic levels ( $\mathrm{n}=7$, each group)

The third protocol was established to determine the effects of a hot shot and reperfusion with EHNA/NBMPR on myocardial purine release and recovery of ventricular performance in a model of 20 minutes of normothermic aortic crossclamping, continuous infusion of retrograde coronary sinus cardioplegia, and reperfusion. Ischemic hearts were infused with $500 \mathrm{~mL}$ hot shot cardioplegic solution $\left(37^{\circ} \mathrm{C}\right)$ with either the vehicle solution ( $\mathrm{n}=8$ ) or vehicle solution containing $100 \mu \mathrm{M}$ EHNA and $25 \mu \mathrm{M}$ NBMPR $(\mathrm{n}=8)$ before switching to continuous infusion of hypothermic $\left(4^{\circ} \mathrm{C}\right)$ cardioplegic solution with a maximal pressure of $40 \mathrm{~mm} \mathrm{Hg}(20-25 \mathrm{~mL} / \mathrm{min}$ ute). The rationale of using a hot shot of cardioplegic solution as a mean for drug delivery is that progressive cooling induces a phase transition in cellular membrane phospholipids in such a way that they become rigid and impermeable to solutes and solutions. ${ }^{17}$ On the other hand, rapid cooling of beating hearts could be detrimental by triggering intracellular calcium overload that ultimately leads to myocardial contracture (stone hearts). ${ }^{18}$ Myocardial core temperature reached 16 to $18^{\circ} \mathrm{C}$ within 2 to 4 minutes during infusion of cold cardioplegia. At the end of 20 minutes of unprotected ischemia and 100 minutes of cardioplegic arrest, the right atrium was closed via suture. Reperfusion was initiated by releasing the crossclamp and allowing the heart to be reperfused with warm blood with or without EHNA/NBMPR for 60 minutes. Coronary sinus effluent during the first 10 seconds of reperfusion was collected in a $20-\mathrm{mL}$ vial containing 0.1 $\mathrm{mL}$ of a stopping solution of EHNA/NBMPR, then was frozen immediately in liquid nitrogen and kept at $-80^{\circ} \mathrm{C}$ for later extraction and highperformance liquid chromatography (HPLC) analysis. Functional measurements were obtained at baseline, and at 30 minutes and 60 minutes of reperfusion. Myocardial biopsies were obtained at baseline, the end of normothermic aortic crossclamping, during administration of hypothermic cardioplegia, and during reperfusion.

\section{Assessment of Left Ventricular Performance}

The left ventricular performance was assessed, off bypass pump, from the relationship between stroke work and end diastolic dimension as a sensitive and load-independent index of contractility using pulse transit sonomicrometry (Triton Technology, San Diego, Calif) and Millar pressure transducers (Millar Instruments, Houton, Tex) as described previously. ${ }^{2}$ One pair of lead titonate zyrconate-piezoelectric hemispheric crystals was sutured to the anterior and posterior of the epicardial surface of the left ventricle wall in the minor axis $(40-60 \mathrm{~mm})$. Analog data were digitized at $200 \mathrm{~Hz}$ and stored on a personal computer hard disk and analyzed by interactive Crunch software developed in our laboratory. Data acquisition was performed at varying preloads via venous drainage creating diminishing cardiac work loops.

\section{Assessment of Adenine Nucleotide Pool Metabolism}

Transmural Serial Tru-Cut needle (Cardinal Health, Dublin, Ohio) biopsy specimens $(5-10 \mathrm{mg})$ were obtained at baseline, at the end of warm ischemia, during hypothermic arrest, and during reperfusion. Each biopsy was frozen immediately in liquid nitrogen $\left(-196^{\circ} \mathrm{C}\right)$ and later extracted, analyzed, and quantified using HPLC (data are reported as nanomoles per milligram protein). ${ }^{2}$

\section{Statistical Analysis}

Data are presented as mean \pm standard error of the mean. Sequential measurements were compared using repeated-measures analysis of variance and Tukey post hoc tests using SAS (version 6.02; Statistical Analysis System Institute, Cary, NC). Differences were considered significant if the probability value for comparison of least squares means was $<.05$.

\section{RESULTS}

\section{Effects of Infusion of Intermittent Antegrade or Continuous Coronary Sinus Retrograde Hypothermic Cardioplegia on Levels of Myocardial Purines and Their Release From Acutely Ischemic Hearts}

Twenty minutes of on-pump normothermic aortic crossclamping depleted myocardial ATP by about $20 \%$ to $35 \%$ and resulted in a concomitant accumulation of mainly myocardial inosine (Table 1). Induction of either intermittent antegrade or continuous coronary sinus hypothermic cardioplegia was associated with early (1-4 minutes) release of inosine, hypoxanthine, and xanthine, all of which are known to be sensitive markers of ischemia. Purine release was abolished when myocardial temperature reached the cooling range $\left(16-18^{\circ} \mathrm{C}\right)$ within 1 to 4 minutes (Figure 1 , $A$ and $B$, and Figure E2, $A$ ). This observation was not only noted with the initial induction of antegrade cardioplegia $(1000 \mathrm{~mL})$, but also with each subsequent bolus $(500$ $\mathrm{mL}$ ) of intermittent antegrade cardioplegia every $20 \mathrm{~min}$ utes. On the other hand, induction of continuous coronary sinus cardioplegia was associated with release of purines into blood effluent collected from the aortic cannula within 1 to 4 minutes of induction of cardioplegia. Purine release ceased when myocardial temperature reached $<20^{\circ} \mathrm{C}$ (baseline levels) (Figure E2, B). Reperfusion with warm, oxygenated blood robustly released and converted the remaining purines in the ischemic myocardium to hypoxanthine and xanthine (Figure 1, $A$ and $B$, and Table 2). 

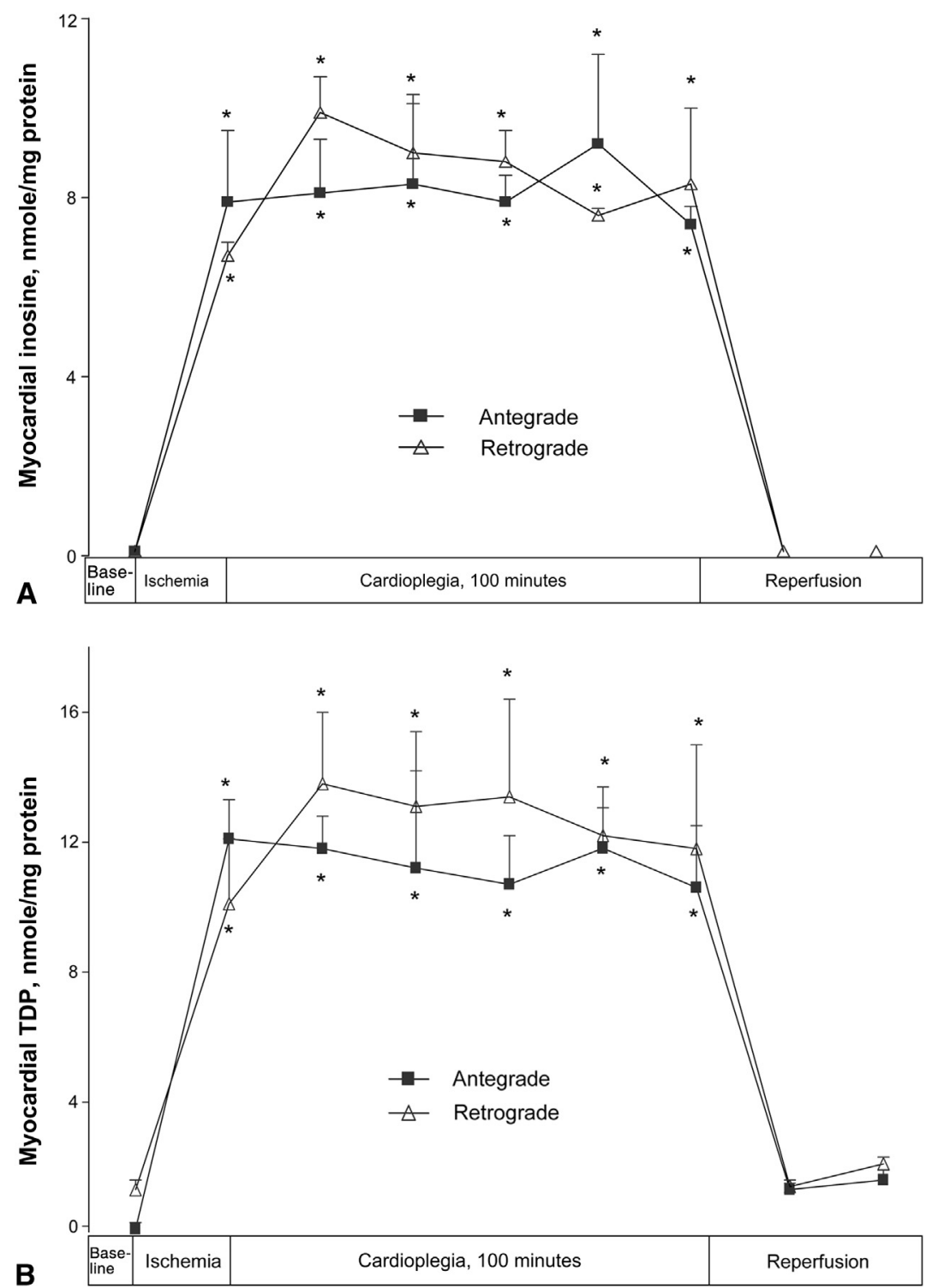

FIGURE 1. Effects of intermittent antegrade or continuous retrograde coronary sinus hypothermic cardioplegia on the levels of myocardial purines as markers of ischemia of the left ventricle in acutely ischemic hearts. All hearts were subjected to 20 minutes of warm aortic crossclamping on-pump followed 100 minutes of hypothermic cardioplegic arrest and 60 minutes of reperfusion via antegrade or retrograde routes. The $P$ value was not significant among groups using analysis of variance. $* P<.05$ versus baseline levels $(\mathrm{n}=7)$. A, Effects of intermittent antegrade or continuous retrograde coronary sinus hypothermic cardioplegia on myocardial inosine levels of the left ventricle in acutely ischemic hearts. B, Effects of intermittent antegrade or continuous retrograde coronary sinus hypothermic cardioplegia on myocardial total diffusible purine (adenosine + inosine + hypoxanthine + xanthine) levels of the left ventricle in acutely ischemic hearts. $T D P$, Total diffusible purines.

\section{Effects of Postischemic Reperfusion With EHNA/}

NBMPR on the Levels of Myocardial Purines and Left Ventricular Performance in Acutely Ischemic Hearts Without Cold Cardioplegic Arrest

The efficacy of postischemic administration of EHNA/ NBMPR was previously reported ${ }^{8}$ in the same canine model and results were used here for comparison. Unprotected aortic crossclamping caused about $25 \%$ to $35 \%$ depletion of myocardial ATP with a concomitant accumulation of mainly inosine (Table 3). Reperfusion was initiated by allowing the heart to be reperfused with warm blood perfusion containing either the vehicle solution or $100 \mu \mathrm{M}$ EHNA and $25 \mu \mathrm{M}$ NBMPR. In the control group, ventricular performance was depressed by about $50 \%$ compared with baseline function. The slope of the relationship between stroke work/end diastolic length declined significantly to $40.1 \pm 6 \mathrm{dyne} / \mathrm{cm}^{2} \cdot 10^{3}$ and $51.3 \pm 3$ dyne $/ \mathrm{cm}^{2} \cdot 10^{3}$ at 30 minutes and 60 minutes of reperfusion, respectively $(P<.05$ vs baseline function, $103.5 \pm 4$ dyne/ $\mathrm{cm}^{2} \cdot 10^{3}$; Table 3 ). A complete recovery of ventricular 
TABLE 2. Purine release at the induction of intermittent antegrade or continuous retrograde hypothermic cardioplegia in acutely ischemic hearts and reperfusion

\begin{tabular}{lccccc}
\hline & \multicolumn{2}{c}{ Percent of total diffusible purines } \\
\cline { 2 - 3 } Metabolites & \multicolumn{2}{c}{ Antegrade } & & \multicolumn{2}{c}{ Retrograde } \\
\cline { 2 - 5 } \cline { 5 - 6 } & Induction of cardioplegia & Induction of reperfusion & & Induction of cardioplegia & Induction of reperfusion \\
\hline Adenosine & $1.30 \pm 0.6$ & $0.31 \pm 0.2$ & & $0.07 \pm 0.1$ & $0.73 \pm 0.6$ \\
Inosine & $17.12 \pm 7.3^{*}$ & $2.17 \pm 0.3^{*}$ & & $11.66 \pm 6.1^{*}$ & $2.18 \pm 1.3^{*}$ \\
Hypoxanthine & $53.38 \pm 8.6^{*}$ & $49.50 \pm 8.2^{*}$ & & $54.97 \pm 13^{*}$ & $50.84 \pm 8.5^{*}$ \\
Xanthine & $19.94 \pm 8.1^{*}$ & $44.03 \pm 7.4^{*}$ & & $29.35 \pm 10^{*}$ & $41.00 \pm 6.4^{*}$ \\
\hline
\end{tabular}

Baseline levels of purines in plasma of arterial and venous blood from the coronary sinus were below detection levels. On-pump canine hearts were subjected to 20 minutes of normothermic aortic crossclamping followed by induction of either antegrade or retrograde hypothermic cardioplegia. The initial coronary sinus effluents were collected within the first 10 seconds into a vial containing a stopping solution (erythro-9-[2-hydroxy-3-nonyl] adenine/p-nitro-benzylthioinosine), then were frozen, extracted, and analyzed by high-performance liquid chromatography. Results are presented as a percentage of content in milliliters of collected plasma in each study, and reflect the release and conversion of myocardial adenine nucleosides to hypoxanthine and xanthine. $* P<.05$ versus adenosine levels ( $\mathrm{n}=7$, each group).

function was observed in the group that was reperfused with warm blood containing EHNA/NBMPR. The slopes of stroke work/end diastolic length relationship in the latter group reached $108.2 \pm 6 \mathrm{dyne} / \mathrm{cm}^{2} \cdot 10^{3}$ and $93.7 \pm 5$ dyne $/ \mathrm{cm}^{2} \cdot 10^{3}$ at 30 minutes and 60 minutes of reperfusion, respectively ( $P$-value not significant vs baseline function, $101 \cdot 8 \pm 4$ dyne $/ \mathrm{cm}^{2} \cdot 10^{3}$; Table 3 ).

\section{Effects of a Hot Shot and Reperfusion of EHNA/NBMPR on Cardiac Metabolism and Function in Acutely Ischemic Hearts Undergoing Retrograde Hypothermic Cardioplegic Arrest and Reperfusion}

In this experiment, acutely ischemic hearts were infused with a warm cardioplegic solution (hot shot), containing either the vehicle solution or EHNA/NBMPR, via the coronary sinus prior to switching to retrograde coronary sinus cold cardioplegia. Figure 2 depicts the effects of the coronary sinus shot and reperfusion with EHNA/NBMPR before and after continuous retrograde coronary sinus hypothermic cardioplegia on myocardial ATP, adenosine, inosine, and total diffusible purines. The effects of hot shot and reperfusion with EHNA/NBMPR before and after continuous retrograde coronary sinus hypothermic cardioplegia, respectively, on myocardial ATP in acutely ischemic are shown in Figure 2, A. Warm aortic crossclamping caused similar depletion of myocardial ATP in the control and drug-treated groups $(P<.05$ vs baseline $)$. Myocardial ATP levels were higher during cardioplegic arrest compared with ATP levels at the end of warm ischemia and after reperfusion. The reasons for these differences are not known; however, they could be related to the fact that myocardial biopsies obtained during cardioplegic arrest had less red blood cell contaminant, thus reducing protein values in those particular biopsies on which ATP levels are normalized. The levels of myocardial adenosine (Figure 2, B) and inosine (Figure 2,C) as well as total diffusible purines (Figure 2,D) increased significantly during warm ischemia

TABLE 3. Effects of postischemic reperfusion with EHNA/NBMPR on left ventricular performance and purine metabolism in acutely ischemic hearts without hypothermic cardioplegic arrest

\begin{tabular}{|c|c|c|c|c|c|c|}
\hline \multirow[b]{2}{*}{ Parameters } & \multirow[b]{2}{*}{ Baseline } & \multirow[b]{2}{*}{ Ischemia, $20 \mathrm{~min}$} & \multicolumn{4}{|c|}{ Reperfusion } \\
\hline & & & $30 \mathrm{~min}$ & $60 \mathrm{~min}$ & $90 \mathrm{~min}$ & $120 \mathrm{~min}$ \\
\hline \multicolumn{7}{|l|}{ LV performance } \\
\hline Control group $(\mathrm{n}=8)$ & $103.5 \pm 4$ & - & $40.1 \pm 6^{*}$ & $51.3 \pm 3 *$ & $60.8 \pm 5^{*}$ & $59.8 \pm 3 *$ \\
\hline EHNA/NBMPR group & $101.8 \pm 4$ & & $108.2 \pm 6 \dagger$ & $93.7 \pm 5 \dagger$ & $93.5 \pm 6 \dagger$ & $95.4 \pm 4 \dagger$ \\
\hline \multicolumn{7}{|l|}{ Adenosine triphosphate } \\
\hline Control group & $24.2 \pm 2$ & $18.2 \pm 2 *$ & $17.6 \pm 7^{*}$ & $22.0 \pm 2 *$ & $18.1 \pm 3^{*}$ & $17.9 \pm 2 *$ \\
\hline EHNA/NBMPR group & $27.3 \pm 2$ & $17.5 \pm 3 *$ & $17.2 \pm 2 *$ & $17.1 \pm 6^{*}$ & $16.3 \pm 2 *$ & $16.9 \pm 2 *$ \\
\hline \multicolumn{7}{|l|}{ Adenosine } \\
\hline Control group & $0.10 \pm 0.1$ & $1.90 \pm 0.4^{*}$ & $0.20 \pm 0.1$ & $0.10 \pm 0.1$ & $0.10 \pm 0.1$ & $0.10 \pm 0.1$ \\
\hline EHNA/NBMPR group & $0.10 \pm 0.1$ & $1.75 \pm 0.2^{*}$ & $0.49 \pm 0.2$ & $0.25 \pm 0.1$ & $0.26 \pm 0.1$ & $0.2 \pm 0.1$ \\
\hline \multicolumn{7}{|l|}{ Inosine } \\
\hline Control group & $0.10 \pm 0.1$ & $9.80 \pm 0.3^{*}$ & $1.00 \pm 0.2^{*}$ & $0.14 \pm 0.1$ & $0.10 \pm 0.1$ & $0.10 \pm 0.1$ \\
\hline EHNA/NBMPR group & $0.10 \pm 0.1$ & $7.40 \pm 0.4^{*}$ & $3.60 \pm 0.3^{*}, \dagger$ & $1.43 \pm 0.1 *, \dagger$ & $0.10 \pm 0.1$ & $0.10 \pm 0.1$ \\
\hline
\end{tabular}



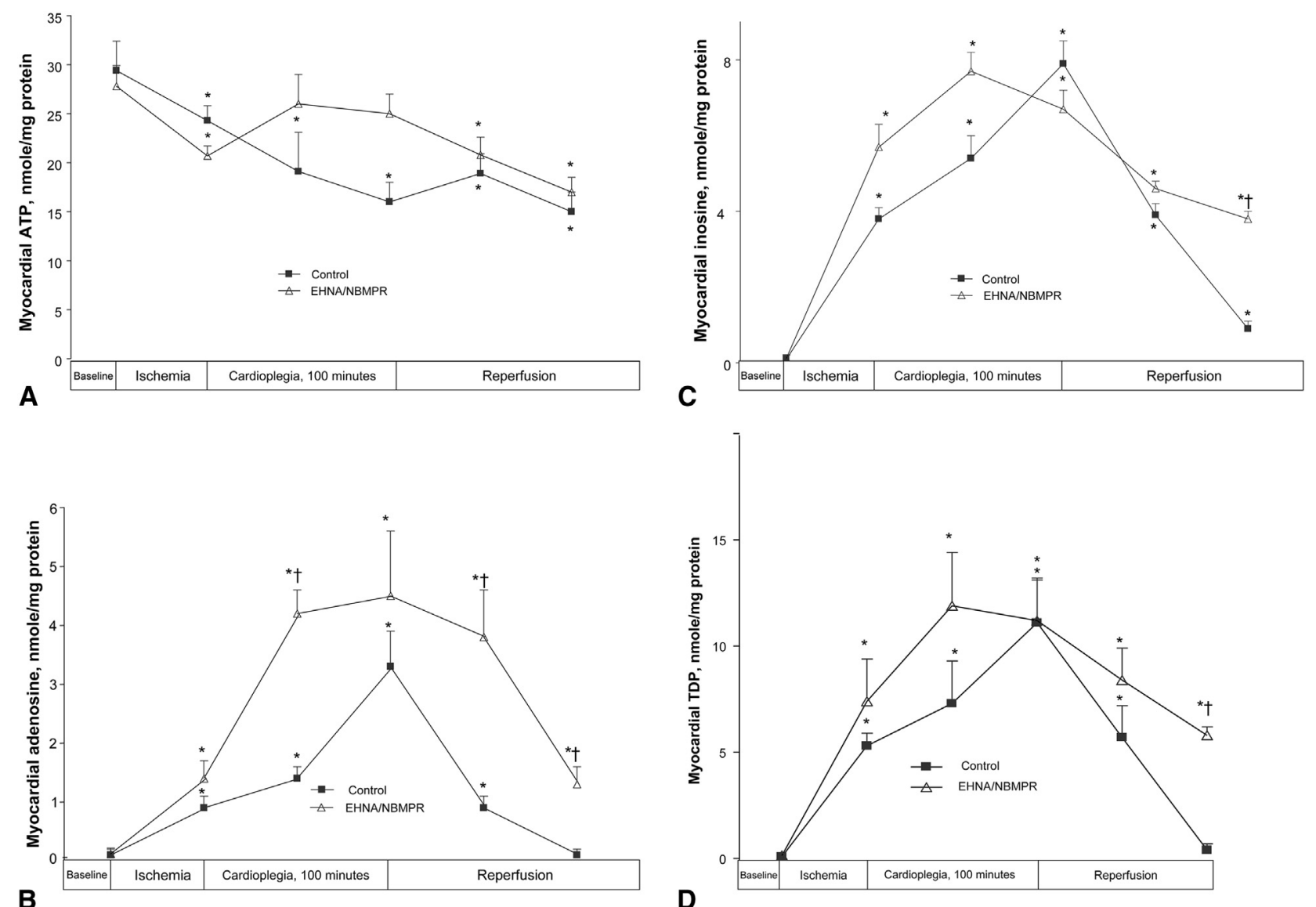

FIGURE 2. Effects of a coronary sinus hot shot and reperfusion with EHNA/NBMPR before and after continuous retrograde coronary sinus hypothermic cardioplegia on myocardial ATP pool metabolism of the left ventricle in acutely ischemic hearts. All hearts were subjected to 20 minutes of warm aortic crossclamping on-pump, a hot shot with or without EHNA/NBMPR, followed 100 minutes of retrograde hypothermic cardioplegic arrest and 60 minutes of reperfusion with or without inhibitors. The $P$ value was not significant among groups using analysis of variance. $* P<.05$ versus baseline ( $\mathrm{n}=8$, each group). $\dagger P<.05$ between groups $(\mathrm{n}=8)$. A, Effects of a coronary sinus hot shot and reperfusion with EHNA/NBMPR before and after continuous retrograde coronary sinus hypothermic cardioplegia on myocardial ATP of the left ventricle in acutely ischemic hearts. B, Effects of a hot shot and reperfusion with EHNA/ NBMPR before and after continuous retrograde coronary sinus hypothermic cardioplegia on myocardial adenosine of the left ventricle in acutely ischemic hearts. C, Effects of a hot shot and reperfusion with EHNA/NBMPR, before and after continuous retrograde coronary sinus hypothermic cardioplegia, on myocardial inosine of the left ventricle in acutely ischemic hearts. D, Effects of a hot shot and reperfusion with EHNA/NBMPR before and after continuous retrograde coronary sinus hypothermic cardioplegia on myocardial total diffusible purines (adenosine + inosine + hypoxanthine + xanthine) of the left ventricle in acutely ischemic hearts. ATP, Adenosine triphosphate; EHNA, erythro-9-[2-hydroxy-3-nonyl] adenine; NBMPR, p-nitro-benzylthioinosine; $T D P$, total diffusible purines.

and remained elevated during the administration of retrograde cardioplegia. Reperfusion triggered a massive loss of myocardial adenosine, inosine, and total diffusible purines in the control group, reflecting their release. Significant levels of adenosine, inosine, and total diffusible purines remained in hearts treated with EHNA/NBMPR as a hot shot and during reperfusion $(P<.05$ vs control; Figure $2, A$ and $B$ ).

Twenty minutes of aortic crossclamping depleted myocardial ATP and accumulated more inosine than adenosine in the right ventricle, similar to that of the left ventricle (Figure E3, $A-D$ ). Infusion of continuous retrograde cardioplegia retained myocardial purines in the right ventricle after hot shot with or without EHNA/NBMPR. Reperfusion after 100 minutes of hypothermic arrest triggered a marked loss of myocardial purines from the right ventricle, similar to that of the left ventricle (Figure E3, $A-D$ ).

Cardiac function was depressed in the control group to about $35 \%$ of baseline function. Left ventricle performance declined to $30.0 \pm 7 \mathrm{dyne} / \mathrm{cm}^{2} \cdot 10^{3}$ and $36.0 \pm 6 \mathrm{dyne} /$ $\mathrm{cm}^{2} \cdot 10^{3}$ at 30 minutes and 60 minutes reperfusion after cardioplegic arrest, respectively $(P<.05$ vs baseline function, $85.0 \pm 3 \mathrm{dyne} / \mathrm{cm}^{2} \cdot 10^{3}$ ). In contrast, a significant recovery of cardiac function was observed the treated group with EHNA/NBMPR as a hot shot before cold cardioplegia and was also reperfused with warm blood containing drugs (Figure 3, A). Recovery of cardiac function at 30 minutes and 60 minutes of reperfusion after cardioplegic arrest 


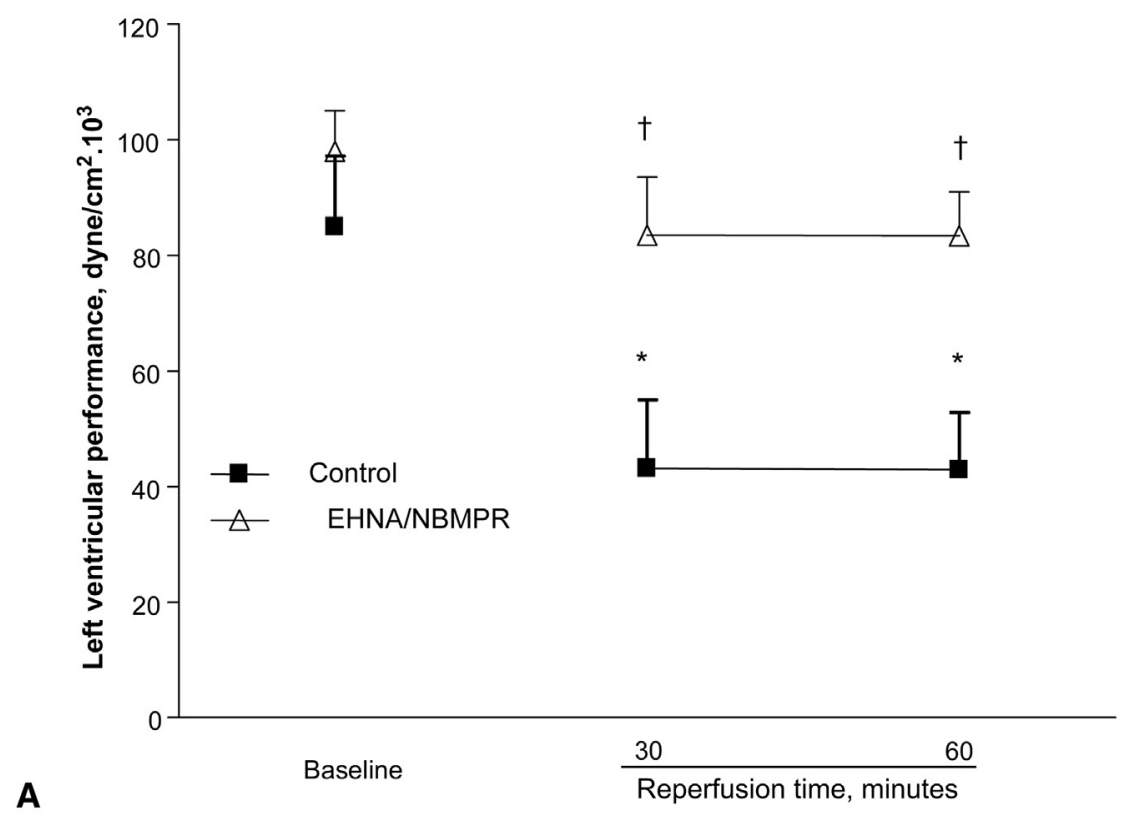

\section{A}

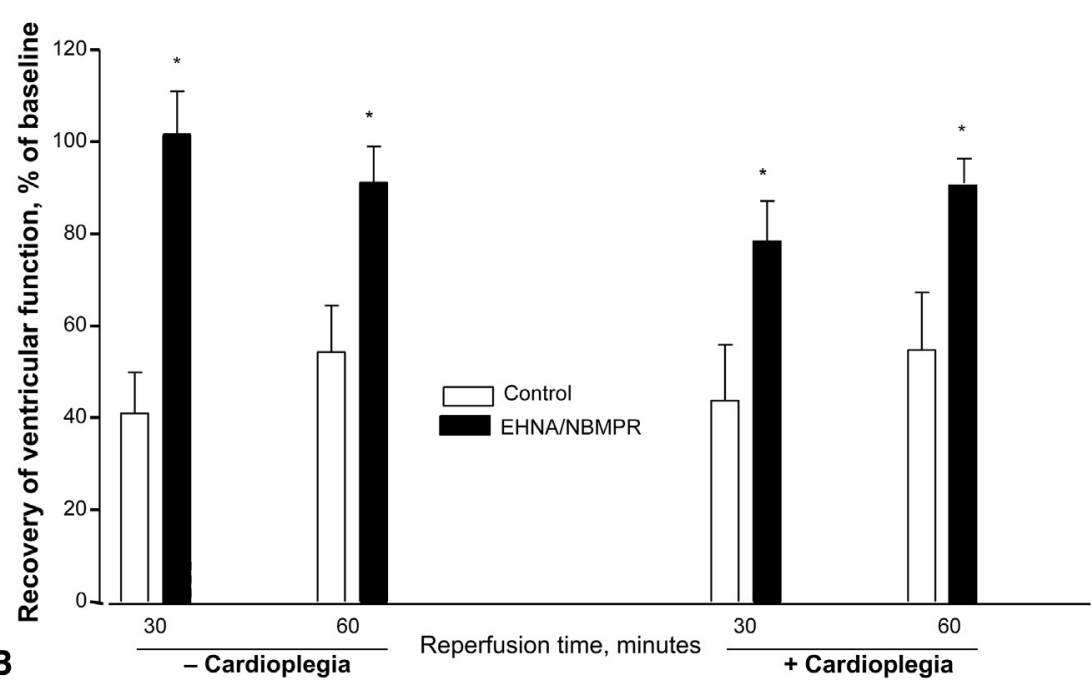

FIGURE 3. Postischemic inhibition of es-ENT1 abolishes ventricular dysfunction induced by an acute warm aortic crossclamping with or without a period of 100 minutes of hypothermic cardioplegic arrest. A, Effects of a hot shot and reperfusion with EHNA/NBMPR, before and after continuous retrograde coronary sinus hypothermic cardioplegia on postischemic recovery of ventricular performance in a canine model of ischemia and reperfusion. The $P$ value was not significant among groups using analysis of variance. ${ }^{*} P<.05$ versus baseline and control group. $\nmid P<.05$ between groups (n $=8$ ). B, Effects of postischemic reperfusion with EHNA/NBMPR on cardiac functional recovery are summarized in the left and right panels. Left panel, Recovery of heart performance after normothermic aortic crossclamping and reperfusion with warm blood, without or with EHNA/NBMPR, in the absence of hypothermic cardioplegic arrest. Preischemic values of ventricular performance in this model of warm ischemia and reperfusion were $103.5 \pm 4 \mathrm{dyne} / \mathrm{cm}^{2} \cdot 10^{3}$ and $101 \pm 4 \mathrm{dyne} / \mathrm{cm}^{2} \cdot 10^{3}$ in the control and EHNA/NBMPR-treated groups, respectively. Right panel, Recovery of heart performance after normothermic aortic cross-clamping, EHNA/NBMPR hot shot, 100 minutes of continuous retrograde cardioplegia, and reperfusion without and with EHNA/NBMPR. Preischemic values of ventricular performance in this model of warm ischemia and reperfusion were $85.0 \pm 3 \mathrm{dyne} / \mathrm{cm}^{2} \cdot 10^{3}$ and $76.3 \pm 6 \mathrm{dyne} /$ $\mathrm{cm}^{2} \cdot 10^{3}$ in the control hot shot and in EHNA/NBMPR hot shot groups, respectively. $* P<.05$ versus the control group, analysis of variance ( $\mathrm{n}=8$, each group). EHNA, Erythro-9-[2-hydroxy-3-nonyl] adenine; NBMPR, p-nitro-benzylthioinosine.

was $59.0 \pm 3 \mathrm{dyne} / \mathrm{cm}^{2} \cdot 10^{3}$ and $77.0 \pm 3 \mathrm{dyne} / \mathrm{cm}^{2} \cdot 10^{3}$, respectively $(P$-value not significant vs baseline function, $76.3 \pm 6$ dyne $/ \mathrm{cm}^{2} \cdot 10^{3}$ ). Figure $3, B$ summarizes the efficacy of EHNA/NBMPR intervention in acutely ischemic hearts with (Figure 3, B, right panel) or without
(Figure 3, B, left panel) hypothermic cardioplegic arrest. Results demonstrated a full recovery of cardiac function (90\%-100\%) when ischemic hearts were reperfused with drugs containing blood without cold cardioplegic arrest. Significant recovery of left ventricular performance 
(80\%-90\%) was observed when hearts were infused with an EHNA/NBMPR-containing hot shot before hypothermic cardioplegia and then followed by reperfusion with EHNA/ NBMPR containing blood. Despite recovery of cardiac function, the levels of myocardial ATP remained depressed to the levels at the end of 20 minutes of warm ischemia and were not improved by an additional 100 minutes of hypothermic cardioplegic arrest on-pump. These results demonstrate that acutely ischemic hearts are well protected during hypothermic cardioplegic arrest.

\section{DISCUSSION}

The rationale of this investigation was that myocardial purines released from acutely ischemic hearts via the esENT1 nucleoside transporter play a major role in the early events of reperfusion injury. ${ }^{2,4}$ Myocardial purines are considered to be sensitive markers of acute ischemia and are also known to serve as specific substrates of oxygenderived free radicals on reperfusion. Under normothermia, pharmacologic modulation of purine release from ischemic myocardium by inhibiting the es-ENT1 nucleoside transporter proved to be an effective strategy in attenuating purine-mediated reperfusion injury endpoints such as ventricular dysfunction, myocardial stunning, ${ }^{2,13}$ and fibrillation. ${ }^{9,18}$ In the current study, the efficacy of EHNA/ NBMPR in a clinically relevant model has been challenged with infusion of hypothermic cardioplegia.

Results from this investigation demonstrated that induction of hypothermic cardioplegia triggers an early release of (1-4 minutes) of purines from ischemic hearts. However, purine release stopped completely when the temperature of the myocardium became cold $\left(<20^{\circ} \mathrm{C}\right)$ within 2 to 4 minutes induction of cardioplegia (Figure E2, $A$ and $B$ ). This observation was not only noted on the first induction bolus of antegrade cardioplegia $(1000 \mathrm{~mL})$, but also was observed with each consecutive bolus of intermittent antegrade cardioplegia (500 mL every 20 minutes). Induction of continuous retrograde coronary sinus hypothermic cardioplegia was also associated with of release of purines (collected from the aortic cannula) within 1 to 4 minutes, then declined progressively to precardioplegia baseline levels after 4 minutes, when myocardial temperature reached $<20^{\circ} \mathrm{C}$. Reperfusion with warm blood triggered total loss of myocardial purines from cold arrested hearts (Figure 1, $A$ and $B$, and Table 2) in both intermittent antegrade and continuous retrograde hypothermic cardioplegia. It was also interesting to note that although early induction of either intermittent or continuous cold cardioplegia was associated with partial release of purines in the decanted effluents, the ischemic myocardium retained most of the inosine and other diffusible purines (total diffusible purines) during the course of administration (100 minutes) of intermittent antegrade or continuous retrograde hypothermic cardioplegia (Figure 2, $A$ and $B$ ) until reperfusion with warm blood. These observations suggest that cellular membranes have lost their ability to diffuse or transport purines when myocardial temperature reaches $<20^{\circ} \mathrm{C}$. In this respect, there were no differences between intermittent antegrade and continuous retrograde hypothermic cardioplegia with respect to entrapment of purine during cold cardioplegic arrest. Indeed, progressive hypothermia changes cellular membrane fluidity states from liquid to gel to solid, during which the membranes become impermeable to water and solutes, ${ }^{17}$ and this may have caused configurational changes in membrane-bound proteins and loss of their physiologic function. It is interesting to note that in the absence of cold cardioplegia, reperfusion of acutely ischemic hearts with warm blood containing EHHNA/ NBMPR also inhibited purine loss from the ischemic myocardium during reperfusion and abolished the ventricular dysfunction observed in the untreated control group (Table 3). Considering all our results, specific modulation of the es-ENT1 nucleoside transporter before or after warm ischemia, or even with or without prolonged hypothermic cardioplegic arrest, is believed to be an effective strategy of myocardial protection in experimental models that simulate the clinical practice of cardiac surgery. However, it is not known whether inhibition of the es-ENT1 nucleoside transporter by EHNA/NBMPR, per se, provides other unknown metabolic mechanisms of protection beyond that of blockade of purine release and stimulation of free radical pathways on reperfusion.

\section{Hot Shot Drug Delivery}

The rationale for using a hot shot as the delivery system of EHNA/NBMPR is that cold cardioplegia prevents purine release as a result of changes in membrane fluidity, and that drug delivery at normothermia in a depolarized heart would be more effective than at hypothermia. In the current study, a terminal hot shot was replaced with warm blood reperfusion with EHNA/NBMPR, without hyperkalemia, at the end of 20 minutes of warm aortic crossclamping (warm onpump surgery) or at the end of 100 minutes of hypothermic cardioplegic arrest (hypothermic on-pump surgery). Administration of a cardioprotective agent as an adjuvant to cold cardioplegia raises serious questions regarding the efficiency of drug delivery, doses, and efficacy in clinical trials when hypothermic cardioplegic arrest is used as a procedure of choice for myocardial protection. Indeed, experimental studies and clinical trials using adjuvant agents with hypothermia have overlooked the changes in membrane biophysics, including membrane fluidity and permeability, thus underscoring a drug's pharmacologic efficacy. For example, previous experimental studies have demonstrated that preischemic treatment with low doses of adenosine (100 $\mu \mathrm{M}$ or less) exerted powerful cardioprotection in animal models of acute myocardial infarcation. ${ }^{19}$ An early clinical trial with adenosine has demonstrated a lack of cardioprotective efficacy of adenosine when used as an 
adjuvant drug to hypothermic cardioplegia ${ }^{20}$ at 5 different doses between $100 \mu \mathrm{M}$ and $2 \mathrm{mM}$. These results demonstrated that lower requirement of dopamine, but not reduction in infarct size, was observed in the group of patients who received the highest dose of adenosine $(2 \mathrm{mM})$ with additional preischemic adenosine (140 $\mu \mathrm{g} / \mathrm{kg} /$ minute). In a subsequent trial, 2 doses of adenosine were used (ie, $500 \mu \mathrm{M}$ and $2 \mathrm{mM}$ ) along with supplemental adenosine during reperfusion of $200 \mathrm{mg}$ adenosine $/ \mathrm{kg} /$ minute for $10 \mathrm{~min}$ utes before and 15 minutes after releasing the aortic crossclamp. The only favorable outcome was a reduced need for inotropic support as noted in patients who received only $2 \mathrm{mM}$ adenosine with an additional adenosine supplement. ${ }^{21}$ Lack of pharmacologic efficacy of adenosine when used in cold cardioplegia could be related to lack of drug delivery resulting from changes in membrane physical properties. ${ }^{17}$ Whether adenosine would be more efficacious under normothermic or tepid temperatures than hypothermic conditions remains to be demonstrated in future clinical trials.

It is anticipated that postischemic warm delivery of EHNA/NBMPR maximizes pharmacologic efficacy at normothermia before and after hypothermic cardioplegic arrest. Introducing EHNA/NBMPR into the cardiopulmonary bypass prior to reperfusion was effective in attenuating ventricular dysfunction of acutely ischemic hearts under both normothermic and hypothermic experiments. Results from the current study suggest that attention should be paid to manage reperfusion injury not only at reperfusion, but also during delivery of cardioplegia. Therefore, we are proposing that warm cardioplegia (hot shot) should be considered as a means for drug delivery on induction of cardioplegia in addition to that used as terminal warm blood reperfusion or hot shot after hypothermic cardioplegic arrest in clinical trials.

Pharmacologic intervention with EHNA/NBMPR improved cardiac recovery in a canine model on-pump after $10,20,30$, and 60 minutes of normothermic aortic crossclamping and reperfusion without a period of hypothermic cardioplegic arrest. ${ }^{2,8,9,13,14}$ Nevertheless, warm delivery of EHNA/NBMPR would also be more effective in clinical cases of off-pump beating heart cardiac surgery and during intermittent aortic crossclamping without cardioplegic arrest. In this regard, administration of EHNA/ NBMPR before or after myocardial infarction was effective in attenuating ventricular fibrillation and dysfunction in a canine model of off-pump coronary artery occlusion and reperfusion. ${ }^{18}$ Therefore, myocardial protection for beating heart cardiac surgery off-pump, in the absence of cardiopulmonary bypass and hypothermic cardioplegic arrest, is feasible. Nevertheless, EHNA/NBMPR has been used as a pharmacologic tool to separate the components of ischemic injury from that of reperfusion injury in a canine model of aortic crossclamping and reperfusion. ${ }^{3,4}$ Binding kinetics studies have demonstrated identification of es-ENT1 binding sites in myocardial preparations of different species, including humans. ${ }^{22}$ Gene deletion of es-ENT1 proved to be protective in an isolated mouse heart model of ischemia and reperfusion, ${ }^{23}$ thus supporting our previous ${ }^{2-4,8,9,13-15}$ and current findings that the es-ENT1 nucleoside transporter plays a major role in postischemic reperfusion injury.

\section{Study Limitations}

Although the current study demonstrated the efficacy of EHNA/NBMPR pharmacologic intervention against postischemic reperfusion injury in the settings of warm or cold cardiac surgery, the long-term survival outcome has not been addressed in this study and should be considered in future clinical trials. The dose of EHNA or NBMPR used in the current study was not based on a dose/response curve, but rather was derived from reported Ki values $\left(10^{-9} \mathrm{M}\right)$. Significant retention of purines in the myocardium of the EHNA/NBMPR-treated group suggests that doses of both EHNA and NBMPR were adequate. The issue that is most important-administration of an EHNA/NBMPR hot shot at the time of induction of the hot shot or administration at the time of the reperfusion hot shot-has not been addressed here but warrants further study. The importance of timing, temperature, and the route of administration of cardioprotective drugs must be addressed in clinical trials. In addition, determining and comparing the effects of cold and hot shots with without EHNA/NBMPR is important and should be investigated. Similarly, comparing the effects of EHNA/NBMPR induction and that of EHNA/NBMPR hot shot at reperfusion is also warranted. Other concerns are related to breakdown and metabolism of NBMPR in the liver and the formation of 6-mercaptopurine. The latter compound is known to cause adverse effects when given chronically at high doses in animals. ${ }^{24}$ In our study, the level of NBMPR in blood or plasma was found to be below the sensitivity of HPLC detectors. It is predicted that effects of a single use of EHNA/NBMPR with low concentrations would be negligible and might not have long-term effects. NBMPR is used as a predrug before administration of nucleoside-derived drugs such as antileukemia and malarial, antiretroviral azidothymidine, and anti-HIV/AIDS drugs to protect patients' host cells and, in the meantime, to reduce their pharmacologic doses. ${ }^{25,26}$

\section{CONCLUSIONS}

In acutely ischemic hearts, induction of intermittent antegrade or continuous retrograde hypothermic cardioplegia promotes partial release of myocardial purines from acutely ischemic hearts until myocardial temperatures reach $<20^{\circ} \mathrm{C}$. In contrast, warm blood reperfusion robustly releases purines, causing reperfusion injury. Postischemic reperfusion with EHNA/NBMPR abolished myocardial stunning in the absence of hypothermic cardioplegic arrest. Postischemic hot shots and reperfusion with EHNA/ 
NBMPR also abolished purine-mediated reperfusion injury following prolonged hypothermic cardioplegic arrest. We conclude that es-ENT1 plays a major role in postischemic reperfusion injury in an experimental model simulating intraoperative on-pump cardiac surgery in which hypothermic cardioplegic arrest is used. Targeting postischemic reperfusion injury by inhibiting the es-ENT1 nucleoside transporter is an attractive intervention of intraoperative myocardial protection, especially in high-risk patients and redo cardiac surgery.

\section{References}

1. Braunwald E, Kloner RA. Myocardial reperfusion: a double-edged sword? J Clin Invest. 1985;76:1713-9.

2. Abd-Elfattah AS, Jessen ME, Lekven J, Doherty NE III, Brunsting LA, Wechsler AS. Myocardial reperfusion injury: role of myocardial hypoxanthine and xanthine in free radical-mediated reperfusion injury. Circulation. 1988;78: III224-35.

3. Abd-Elfattah AS, Wechsler AS. Separation between ischemic and reperfusion injury by site-specific entrapment of endogenous adenosine and inosine using NBMPR and EHNA. J Card Surg. 1994;9:387-96.

4. Abd-Elfattah A, Higgins R, Latifi R, Merrell R. Targeting post-ischemic reperfusion injury: scientific cream and clinical reality. New Surg. 2001;1:41-51.

5. Dworkin GH, Abd-Elfattah AS, Yeh T Jr, Wechsler AS. Efficacy of recombinantderived human superoxide dismutase on porcine left ventricular contractility after normothermic global myocardial ischemia and hypothermic cardioplegic arrest. Circulation. 1990;82:IV359-66.

6. Neumann FJ, Ott I, Gawaz M, Richardt G, Holzapfel H, Jochum M, et al. Cardiac release of cytokines and inflammatory responses in acute myocardial infarction. Circulation. 1995;92:748-55.

7. Tofukuji M, Stahl GL, Agah A, Metais C, Simons M, Sellke FW. Anti-C5a monoclonal antibody reduces cardiopulmonary bypass and cardioplegia-induced coronary endothelial dysfunction. J Thorac Cardiovasc Surg. 1998;116:1060-8.

8. Abd-Elfattah AS, Ding M, Dyke CM, Wechsler AS. Protection of the stunned myocardium: selective nucleoside transport blocker administered after $20 \mathrm{~min}$ utes of ischemia augments recovery of ventricular function. Circulation. 1993; 88:II336-43.

9. Abd-Elfattah AS, Ding M, Wechsler AS. Intermittent aortic crossclamping prevents cumulative adenosine triphosphate depletion, ventricular fibrillation, and dysfunction (stunning): is it preconditioning? J Thorac Cardiovasc Surg. 1995; 110:328-39.

10. Abd-Elfattah AS, Al-Watidy AF, Hegab IM, Mohamed A. Ischemic preconditioning of infarcted hearts: role of ATP-sensitive potassium channels. FASEB J. 2011;25:1097.10.

11. Guo JH, Gao SP, Marktanner R, Mahgoub M, Wechsler AS, Abd-Elfattah AS Evidence for chronic preconditioning against myocardial infarction: chronic rapid heart rate induces expression of stress protein 70 (HSP 70) and limits the infarct size: effect of K-ATP channels and A1-receptor blockers. Circulation. 1996;94:I422.
12. Abd-Elfattah AS, Guo J-H, Gao S-P. Failing hearts are preconditioned against myocardial infarction and exhausted their ischemic preconditioning reserve. FASEB J. 2011;25:1033.2.

13. Abd-Elfattah AS, Jessen ME, Hanan SA, Tuchy G, Wechsler AS. Is adenosine 5'- triphosphate derangement or free-radical-mediated injury the major cause of ventricular dysfunction during reperfusion? Role of adenine nucleoside transport in myocardial reperfusion injury. Circulation. 1990;82:IV341-50.

14. Abd-Elfattah AS, Ding M, Jessen ME, Wechsler AS. On-pump inhibition of esENT1 nucleoside transporter and adenosine deaminase during aortic cross clamping entraps $\mathrm{F}$ adenosine and protects against reperfusion injury: role of adenosine A1 receptor. J Thorac Cardiovasc Surg. 2012;144:243-9.

15. Abd-Elfattah AS, Aly H, Hanan S, Wechsler AS. Myocardial protection in beating heart cardiac surgery: I. Pre- or postconditioning with inhibition of es-ENT1 nucleoside transporter and adenosine deaminase attenuates post-MI reperfusionmediated ventricular fibrillation and regional contractile dysfunction. J Thorac Cardiovasc Surg. 2012;144:250-5.

16. US National Institute of Health. Guide for the Care and Use of Laboratory Animals. Washington, DC: National Academies Press; 1996. Available at: http:// www.nap.edu/catalog/5140.html.

17. McMurchie EJ, Raison JK, Cairncross KD. Temperature-induced phase changes in membranes of hearts: a contrast between the thermal response of poikilotherms and homeotherms. Comp Biochem Physiol B Comp Biochem. 1973;44: 1017-26.

18. Rebeyka IM, Hanan SA, Borges MR, Lee KF, Yeh T Jr, Tuchy GE, et al. Rapid cooling contracture of the myocardium: the adverse effect of prearrest cardiac hypothermia. J Thorac Cardiovasc Surg. 1990;100:240-9.

19. Lasley RD, Mentzer RM Jr. Dose-dependent effects of adenosine on interstitial fluid adenosine and postischemic function in the isolated rat heart. J Pharmacol Exp Ther. 1998;286:806-11.

20. Mentzer RM Jr, Rahko PS, Molina-Viamonte V, Canver CC, Chopra PS, Love RB, et al. Safety, tolerance, and efficacy of adenosine as an additive to blood cardioplegia in humans during coronary artery bypass surgery. Am J Cardiol. 1997;79:38-43.

21. Mentzer RM Jr, Birjiniuk V, Khuri S, Lowe JE, Rahko PS, Weisel RD, et al. Adenosine myocardial protection: preliminary results of a phase II clinical trial. Ann Surg. 1999;229:643-9.

22. Abd-Elfattah AS, Hoehner J, Wechsler AS. Identification of nucleoside transport binding sites in the human myocardium. Mol Cell Biochem. 1998;180: 105-10.

23. Rose JB, Naydenova Z, Bang A, Eguchi M, Sweeney G, Choi DS, et al. Equilibrative nucleoside transporter 1 plays an essential role in cardioprotection. Am J Physiol Heart Circ Physiol. 2010;298:H771-7.

24. Grossman AB, Noble AJ, Mamula P, Baldassano RN. Increased dosing requirements for 6-mercaptopurine and azathioprine in inflammatory bowel disease patients six years and younger. Inflamm Bowel Dis. 2008;14:750-5.

25. Gero AM, Scott HV, O'Sullivan WJ, Christopherson RI. Antimalarial action of nitrobenzylthioinosine in combination with purine nucleoside antimetabolites. Mol Biochem Parasitol. 1989;34:87-97.

26. Yao SY, Ng AM, Sundaram M, Cass CE, Baldwin SA, Young JD. Transport of antiviral 3'-deoxy-nucleoside drugs by recombinant human and rat equilibrative, nitrobenzylthioinosine (NBMPR)-insensitive (ENT2) nucleoside transporter proteins produced in Xenopus oocytes. Mol Membr Biol. 2001; 18:161-7. 


\section{Experimental Protocols}

1: Effects of intermittent antegrade or continuous coronary sinus retrograde hypothermic cardioplegia on myocardial purines levels in acutely ischemic hearts

\begin{tabular}{|c|c|c|c|}
\hline Baseline & Ischemia & Cold Cardioplegia (100 minutes) & Reperfusion \\
\hline \multirow{2}{*}{$\begin{array}{c}\text { Intermittent Antegrade } \\
\text { or } \\
\text { Continuous Retrograde }\end{array}$} &
\end{tabular}

2: Effects of post-ischemic reperfusion with EHNA/NBMPR on myocardial purine levels and left ventricular performance in acutely ischemic hearts, without hypothermic cardioplegic arrest

\begin{tabular}{|l|l|l|}
\hline Baseline & Ischemia & Reperfusion (with Vehicle or EHNA/NBMPR) \\
\hline
\end{tabular}

3: Effects of a hot shot and reperfusion with EHNA/NBMPR on myocardial purine levels and left ventricular performance in acutely ischemic hearts undergoing retrograde hypothermic cardioplegic arrest and reperfusion

\begin{tabular}{|l|l|l|l|}
\hline Baseline & Ischemia & Cold Cardioplegia (100 minutes) & Reperfusion (with Vehicle or EHNA/NBMPR) \\
\hline
\end{tabular}

FIGURE E1. Experimental protocols. All animals were heparinized, placed on cardiopulmonary bypass, subjected to 20 minutes of normothermic aortic crossclamping, and divided into the following protocols to determine (1) the effects of intermittent antegrade or continuous coronary sinus retrograde hypothermic cardioplegia on myocardial purine levels in acutely ischemic hearts, (2) the effects of postischemic reperfusion with EHNA/NBMPR on myocardial purine levels and left ventricular performance in acutely ischemic hearts without hypothermic cardioplegic arrest, and (3) the effects of a hot shot and reperfusion with EHNA/NBMPR on myocardial purine levels and left ventricular performance in acutely ischemic hearts undergoing retrograde hypothermic cardioplegic arrest and reperfusion. EHNA, Erythro-9-[2-hydroxy-3-nonyl] adenine; $N B M P R$, p-nitro-benzylthioinosine. 

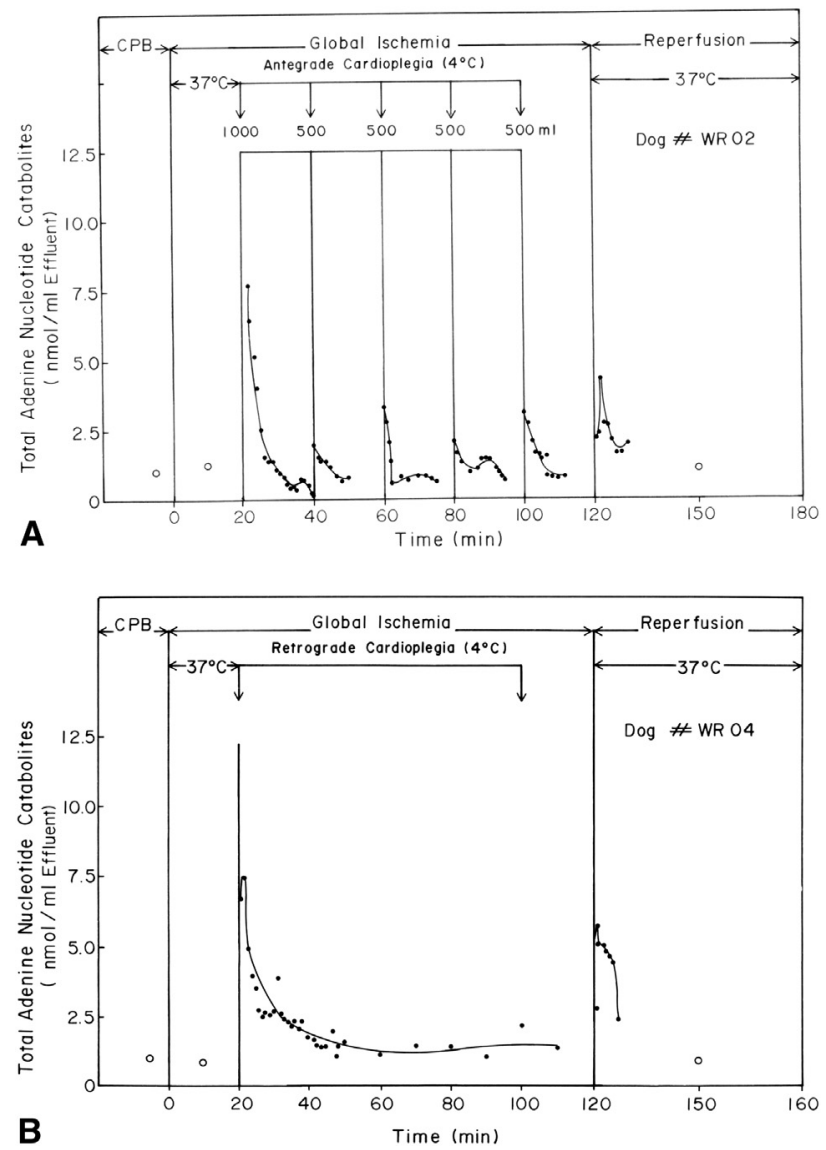

FIGURE E2. A, A typical purine release from an acutely ischemic canine heart (WR 02) during the induction of intermittent antegrade hypothermic cardioplegia via an aortic cannula and measured in the coronary sinus effluents is illustrated. Results demonstrate that the first bolus of antegrade cold cardioplegia was associated with a major release of purines from the ischemic myocardium into the coronary sinus effluent. Purine release was abolished when myocardial temperature was $<20^{\circ} \mathrm{C}$. Administration of each bolus of cardioplegia resulted in a release of purines. Terminal warm blood reperfusion produced a marked peak of purine release (Table 2). B, A typical purine release from an acutely ischemic canine heart (WR 04) during the induction of continuous retrograde coronary sinus hypothermic cardioplegia. Results demonstrated that, while the aortic crossclamp was applied, induction with cold retrograde cardioplegia induced release of purines from the ischemic myocardium into the collected aortic cannula effluent. The release of purines peaked within a few minutes of cardioplegia and declined during hypothermic arrest. Reperfusion triggered a massive release of purines collected from the coronary sinus within 10 seconds of reperfusion (Table 2). $C P B$, Cardiopulmonary bypass. 

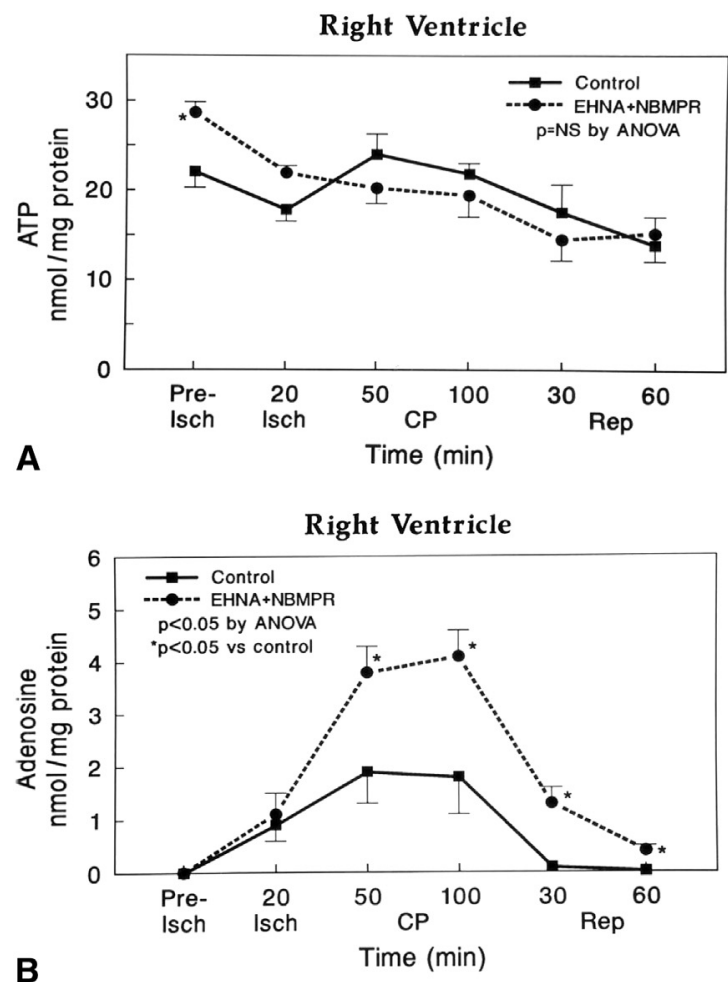

B

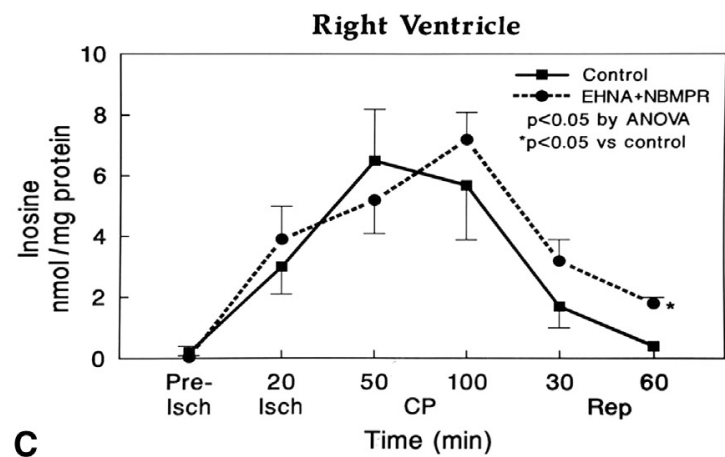

Right Ventricle

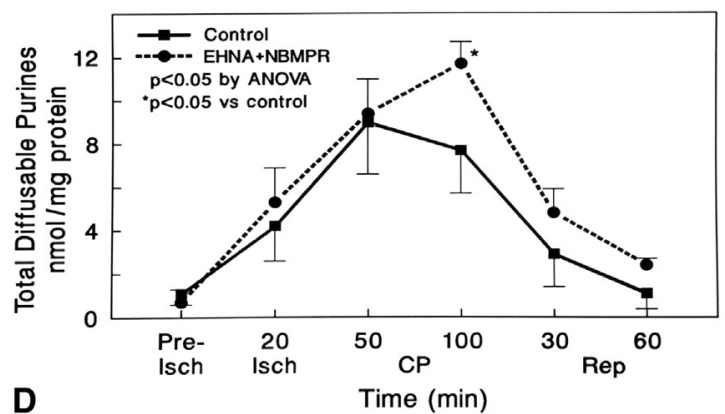

FIGURE E3. Effects of a coronary sinus hot shot and reperfusion with EHNA/NBMPR before and after continuous retrograde coronary sinus hypothermic cardioplegia on myocardial ATP pool metabolism of the right ventricle in acutely ischemic hearts. All hearts were subjected to 20 minutes of warm aortic crossclamping on-pump, a hot shot with or without EHNA/NBMPR, followed 100 minutes of retrograde hypothermic cardioplegic arrest and 60 minutes of reperfusion with or without inhibitors.
$* P<.05$ versus baseline and the control group; $P<.05$ between groups $(\mathrm{n}=8)$. A, Effects of a coronary sinus hot shot and reperfusion with EHNA/NBMPR before and after continuous retrograde coronary sinus hypothermic cardioplegia on myocardial ATP of the right ventricle in acutely ischemic hearts. B, Effects of a hot shot and reperfusion with EHNA/ NBMPR before and after continuous retrograde coronary sinus hypothermic cardioplegia on myocardial adenosine of the right ventricle in acutely ischemic hearts. C, Effects of a hot shot and reperfusion with EHNA/ NBMPR, before and after continuous retrograde coronary sinus hypothermic cardioplegia on myocardial inosine of the right ventricle in acutely ischemic hearts. D, Effects of a hot shot and reperfusion with EHNA/ NBMPR before and after continuous retrograde coronary sinus hypothermic cardioplegia on myocardial total diffusible purines (adenosine + inosine + hypoxanthine + xanthine) of the right ventricle in acutely ischemic hearts. EHNA, Erythro-9-[2-hydroxy-3-nonyl] adenine; $N B M P R$, p-nitro-benzylthioinosine; $A T P$, adenosine triphosphate; $N S$, not significant; $A N O V A$, analysis of variance; $C P$, cardioplegia; Rep, reperfusion. 